\title{
Susceptibilidade diferencial ao herbicida glyphosate e capacidade de rebrota de populações de capim-amargoso ${ }^{1}$
}

\author{
Isadora Fernandes Canedo ${ }^{2}$, Lucas da Silva Araújo ${ }^{3 *} \mathbb{D}$, Luis Gustavo Barroso Silva ${ }^{2}$, Mateus de Souza Valente ${ }^{2}$, \\ Marco Antonio Moreira de Freitas ${ }^{2}$, Paulo César Ribeiro da Cunha ${ }^{2}$
}

10.1590/0034-737X201966010003

\begin{abstract}
RESUMO
Dentre as plantas daninhas com comprovada resistência ao glyphosate, destaca-se o capim-amargoso (Digitaria insularis). $\mathrm{O}$ objetivo deste trabalho foi demonstrar que a susceptibilidade de diferentes populações de $D$. insularis ao glyphosate é consequência do histórico de controle químico adotado nas áreas agrícolas. Sete populações de capimamargoso, provenientes da região produtora de grãos, no sudeste de Goiás (três de Silvânia, duas de Gameleira de Goiás, uma de Pires do Rio e uma de Orizona), foram analisadas por meio da curva dose-resposta. Estudaram-se a susceptibilidade diferencial e a capacidade de rebrota das populações submetidas a diferentes doses de glyphosate $(0 ; 0,11 ; 0,21$; 0,$42 ; 0,84 ; 1,68 ; 3,36 ; 6,72 ; 13,44$ e 26,88 $\mathrm{kg} \mathrm{ha}^{-1}$ i.a.). Além disso, elaborou-se um dendrograma de susceptibilidade ao herbicida, por meio da análise de agrupamento hierárquico UPGMA. As populações de capim-amargoso, manejadas há cinco anos com glyphosate associado a herbicidas inibidores da ACCase, são susceptíveis ao glyphosate; quando manejadas de forma intensiva, unicamente com glyphosate, apresentaram-se menos susceptíveis. O mesmo resultado foi observado com a análise de agrupamento, que indicou a formação de dois grupos, o primeiro composto por populações oriundas de Silvânia, Orizona, Pires do Rio, Gameleira de Goiás, consideradas susceptíveis ao glyphosate e, o segundo grupo, composto por populações oriundas de Gameleira de Goiás e Silvânia, as menos susceptíveis. As populações de capim-amargoso menos sensíveis apresentam maior capacidade de rebrota da parte aérea, após tratadas com doses elevadas de glyphosate.
\end{abstract}

Palavras-chave: controle químico; Digitaria insularis; dose-resposta; resistência.

\section{ABSTRACT}

\section{Differential susceptibility to glyphosate herbicide and re-growth capacity of different populations of sourgrass}

Among the proven weeds proven resistance to glyphosate, the sourgrass (Digitaria insularis) stands out. In this sense, this work was developed with the objective of demonstrating that the susceptibility of different populations of $D$. insularis to glyphosate is a consequence of the chemical control history adopted in the agricultural areas. Seven populations of sourgrass from the southeastern region of Goiás (three from Silvânia, two from Gameleira de Goiás, one from Pires do Rio and one from Orizona) were analyzed using the dose-response curve and the differential susceptibility and the re-growth capacity of the sourgrass populations submitted to different doses of glyphosate $(0,0.11,0.21,0.42$, $0.84,1.68,3.36,6.72,44$ and $26.88 \mathrm{~kg}$ and $\left.\mathrm{ha}^{-1}\right)$. In addition, a herbicide susceptibility dendrogram was elaborated through UPGMA hierarchical cluster analysis. Sourgrass populations, managed five years ago with glyphosate associated with ACCase inhibitor herbicides, are susceptible to glyphosate; when they were treated intensively with glyphosate alone, were less susceptible. The same result was observed for cluster analysis, which indicated the formation of two groups,

Submetido em 14/04/2018 e aprovado em 15/01/2019

1 Este trabalho é parte da dissertação de mestrado do primeiro autor.

2 Instituto Federal Goiano, Departamento de Produção Vegetal, Urutaí, Goiás, Brasil. isadoraknedo@hotmail.com; guga_126@hotmail.com; mateusvalente7@gmail.com; marco.freitas@ifgoiano.edu.br; paulo.cunha@ifgoiano.edu.br

3 Escola Superior de Agricultura Luis de Queiroz, Departamento de Produção Vegetal, Piracicaba, São Paulo, Brasil. 1.s.araujo@usp.br

*Autor para correspondência: 1.s.araujo@usp.br

Rev. Ceres, Viçosa, v. 66, n.1, p. 018-025, jan/fev, 2019 
the first composed by populations from Silvânia, Orizona, Pires do Rio, Gameleira de Goiás conties considered susceptible to glyphosate and the second group composed of populations from Gameleira de Goiás e Silvânia counties, the least susceptible. The less susceptible sourgrass populations have a higher capacity for re-growth after treatment with high doses of glyphosate.

Keywords: Chemical control; Digitaria insularis; dose-response; resistant.

\section{INTRODUÇÃO}

A cultura da soja no Brasil, em 2016/2017, ocupou uma área de 33,91 milhões de hectares (Conab, 2018), com 96,5\% dessa área semeada com cultivares transgênicos, tolerantes a herbicida (Céleres, 2018). Essa predominância da soja tolerante ao glyphosate deve-se a vários fatores, incluindo o amplo espectro de controle das plantas daninhas, baixo custo e a simplicidade de usar apenas um herbicida (Shaner, 2000).

Por essa razão, o glyphosate tornou-se o principal herbicida para a cultura da soja, graças também à seletividade e alta eficácia inicial no controle das plantas daninhas (Green \& Owen, 2011). Entretanto, uma das consequências do uso exclusivo e repetitivo desse herbicida foi a seleção de biótipos de plantas daninhas resistentes.

No Brasil, até 2017, oito espécies foram relatadas com resistência ao glyphosate, com destaque para o capimamargoso (Digitaria insularis) (Heap, 2018). A translocação limitada do herbicida, a degradação rápida do glyphosate e as alterações de dois aminoácidos na enzima EPSPS foram os responsáveis pela resistência dessa espécie daninha ao referido herbicida (Carvalho et al., 2012).

Um estudo de monitoramento da resistência de $D$. insularis nas regiões agrícolas brasileiras, com exceção da região norte, constatou o aumento da frequência e da dispersão de populações resistentes, entre 2012 e 2015 (Lopez Ovejero et al., 2017). Os autores explicam que a rápida disseminação da resistência, de 2012 a 2015, foi consequência das características biológicas da espécie, associadas às práticas agrícolas inadequadas.

Além disso, um dos motivos que agravaram o problema do capim-amargoso foi o potencial de redução da produção de milho e soja, em até 32 e $44 \%$, respectivamente (Gazziero et al., 2012; Gemelli et al., 2013). Portanto, tratase de uma espécie altamente competitiva e de difícil controle após o desenvolvimento de curtos rizomas (Machado et al., 2006).

Machado et al. (2008) caracterizaram o rizoma das plantas de $D$. insularis e observaram a presença de grande quantidade de amido, provavelmente, responsável pela tolerância ao glyphosate, dificultando a translocação e permitindo a rebrota da parte aérea, após tratada com o herbicida. Quando ocorre a perenização (rizoma formado), as plantas produzem elevada quantidade de sementes, revestidas por muitos pelos, facilmente dispersas pelo vento, permitindo a colonização de novos locais (Lorenzi, 2014).

Portanto, a reprodução por sementes e rizomas confere ao capim-amargoso maior potencial de infestação que, somado à evolução da resistência ao glyphosate, contribuiu para o aumento de relatos de dificuldades no controle em diversas áreas agrícolas do Brasil. Nesse sentido, este trabalho teve como hipótese testar a susceptibilidade diferencial ao glyphosate como consequência do histórico do controle químico em populações de capim-amargoso.

Assim, objetivou-se determinar a susceptibilidade de diferentes populações de $D$. insularis ao glyphosate, a capacidade de rebrota das plantas tratadas e discriminar as populações conforme a sensibilidade ao herbicida.

\section{MATERIAL E MÉTODOS}

O experimento foi conduzido em casa de vegetação no Departamento de Produção Vegetal do Instituto Federal Goiano, localizado em Urutaí, Goiás (17²9'4"S; 48¹2’45"O; $725 \mathrm{~m}$ de altitude), no período de setembro de 2015 a março de 2016. As sementes de capim-amargoso foram coletadas em agosto de 2015 (entressafra), em áreas de produção comercial de grãos no sudeste goiano.

O sistema de produção predominante nas áreas agrícolas em que as sementes foram coletadas consiste na sucessão de soja resistente ao glyphosate por milho sem tolerância ao herbicida. Coletaram-se sementes de diferentes plantas (acima de 100 indivíduos) para obter uma amostra representativa da população de cada um dos locais avaliados (Tabela 1).

Os tratamentos foram arranjados em esquema fatorial duplo, com o primeiro fator composto por sete populações de plantas e, o segundo fator, de dez doses crescentes de glyphosate $(0 ; 0,11 ; 0,21 ; 0,42 ; 0,84 ; 1,68 ; 3,36 ; 6,72 ; 13,44$ e $26,88 \mathrm{~kg} i$.a. ha-1). Os vasos foram dispostos em delineamento inteiramente casualizado, com quatro repetições.

A dose discriminatória para elaboração da curva doseresposta foi de $1,68 \mathrm{~kg} \mathrm{ha}^{-1}$ de i.a.. As unidades experimentais foram representadas por vasos de $5 \mathrm{dm}^{-3}$, preenchidos apenas com solo, classificado como Latossolo Vermelho 
distrófico (Embrapa, 2013). O valor de $\mathrm{pH}$ em água foi de 6,$1 ; 0,6 \mathrm{~g} \mathrm{dm}^{-3}$ de matéria orgânica; $1,3 \mathrm{mg} \mathrm{dm}^{-3} \mathrm{de} \mathrm{P} ; 53 \%$ de saturação por bases; $520 \mathrm{~g} \mathrm{~kg}^{-1}$ de areia; $110 \mathrm{~g} \mathrm{~kg}^{-1} \mathrm{de}$ silte; $370 \mathrm{~g} \mathrm{~kg}^{-1}$ de argila.

As sementes foram semeadas em bandejas de isopor preenchidas com substrato comercial à base de fibra de coco, com objetivo de obter um número elevado de mudas. No estádio de crescimento de duas a três folhas totalmente expandidas, as mudas foram transplantadas, mantendo-se a densidade de quatro plantas por vaso. As aplicações das doses de glyphosate foram realizadas no estádio vegetativo de seis perfilhos e altura de $53 \mathrm{~cm}$.

As aplicações foram realizadas no período da manhã, das oito às nove horas, com pulverizador costal pressurizado por $\mathrm{CO}_{2}$ a pressão constante de $3 \mathrm{kgf} \mathrm{cm}^{-2}$, equipado de barra com quatro pontas de pulverização de jato plano Magno ADIA 110.015 , espaçadas de $0,50 \mathrm{~m}$, com volume de calda equivalente a $150 \mathrm{~L} \mathrm{ha}^{-1}$. No momento da aplicação, foram registrados $65 \%$ de umidade relativa do ar, $27^{\circ} \mathrm{C}$ de temperatura ambiente e $4,5 \mathrm{~km} \mathrm{~h}^{-1}$ de velocidade do vento.

Aos 28 dias após a aplicação (DAA), foram realizadas avaliações visuais de controle, utilizando-se escala de notas de 0 a $100 \%$, em que zero representa a ausência de sintomas e $100 \%$ a morte das plantas (SBCPD, 1995).

Para verificar a capacidade de rebrota das plantas coletou-se a parte aérea verde, acima de $5 \mathrm{~cm}$ da superfície do substrato, aos 56 DAA. O material vegetal foi levado para secagem em estufa a $65^{\circ} \mathrm{C}$, até atingir massa constante. Os dados de matéria seca foram expressos em valores percentuais, comparando-se o peso da matéria seca obtida após as aplicações das doses de glyphosate com o peso da matéria seca da testemunha (sem aplicação do produto), considerada $100 \%$.

As curvas de dose-resposta para controle aos 28 DAA e de matéria seca aos 56 DAA foram ajustadas no modelo log-logístico de três parâmetros, como demonstrado por Ritz et al. (2015):

$y=\frac{a}{\left[1+\left(\frac{x}{b}\right)^{c}\right]}$ em que: $y=$ variável resposta; $a=$ amplitude existente entre o ponto máximo e o mínimo da variável; $b=$ dose que promove uma resposta de $50 \%$ da variável; $c=$ inclinação da linha; e $x=$ dose do herbicida $\left(\mathrm{kg} \mathrm{ha}^{-1}\right)$.

Além da dose que corresponde à resposta de $50 \%$ de controle $\left(\mathrm{ED}_{50}\right)$ ou redução da matéria seca $\left(\mathrm{GR}_{50}\right)$, também foi obtida a dose que proporciona a resposta de $80 \%$ de controle $\left(\mathrm{ED}_{80}\right)$ ou redução da matéria seca $\left(\mathrm{GR}_{80}\right)$. A estimativa dos parâmetros $\mathrm{ED}_{80}$ ou $\mathrm{GR}_{80}$ é importante, visto que $80 \%$ constitui o controle mínimo exigido pela legislação em vigor (Carvalho et al., 2005). Assim, foram consideradas populações menos susceptíveis ao herbicida glyphosate, quando apresentarem $\mathrm{ED}_{80}$ ou $\mathrm{GR}_{80}$ acima da dose discriminatória de $1,68 \mathrm{~kg} \mathrm{ha}^{-1}$.

Após a coleta da parte aérea, os vasos foram mantidos sob irrigação periódica controlada, com intuito de verificar a capacidade de rebrota das sete populações, aos 56 dias após o corte (DAC). Para isso, atribuíram-se os valores de 0 e 1 , em que zero representa a ausência e 1 a presença da rebrota, respectivamente. A curva de dose-resposta para a rebrota foi ajustada por modelo log-logístico de dois parâmetros para dados binominais, de acordo com Ritz et al. (2015):

$y=\frac{1}{\left[1+\left(\frac{x}{b}\right)\right]}$

em que: $y=$ proporção de rebrota; $x=$ dose do herbicida $\left(\mathrm{kg} \mathrm{ha}^{-1}\right)$; e $b=$ dose que promove uma resposta de $50 \%$ da variável resposta.

Para verificar a qualidade do ajuste dos dados (controle, matéria seca e rebrota) aos modelos log-logísticos, efetuou-se o teste "lack of fit", que compara os resíduos da regressão não linear com os resíduos da ANOVA (fatorial). Logo, quando o teste for não significativo ( $p>0,05)$, isso indica que os modelos log-logísticos propostos são adequados e, portanto devem ser utilizados para análise dos dados (Ritz et al., 2015).

Além disso, com os parâmetros $\mathrm{C}_{50}, \mathrm{C}_{80}, \mathrm{GR}_{50}$ e $\mathrm{GR}_{80}$ obtidos por meio da curva dose-resposta, elaborou-se

Tabela 1: Coordenadas geográficas dos diferentes locais de coleta na entressafra, agosto de 2015 e, histórico de controle químico das populações de capim-amargoso

\begin{tabular}{|c|c|c|c|c|}
\hline População & Coordenadas & Altitude(m) & Município de Goiás & Controle químico $^{1 /}$ \\
\hline POP1 & S $16^{\circ} 36^{\prime} 30^{\prime \prime} \mathrm{W} 48^{\circ} 37^{\prime} 37^{\prime \prime}$ & 962 & Silvânia & EPSPS/ACCase \\
\hline POP2 & S $17^{\circ} 10^{\prime} 59^{\prime \prime} \mathrm{W} 48^{\circ} 18^{\prime} 44^{\prime \prime}$ & 867 & Orizona & EPSPS/ACCase \\
\hline POP3 & S $16^{\circ} 26^{\prime} 29^{\prime \prime} \mathrm{W} 48^{\circ} 39^{\prime} 27^{\prime \prime}$ & 1054 & Gameleira de Goiás & EPSPS \\
\hline POP4 & S $17^{\circ} 06^{\prime} 13^{\prime \prime} \mathrm{W} 48^{\circ} 24^{\prime} 54^{\prime \prime}$ & 910 & Pires do Rio & EPSPS/ACCase \\
\hline POP5 & $\mathrm{S} 16^{\circ} 37^{\prime} 28^{\prime \prime} \mathrm{W} 48^{\circ} 38^{\prime} 25^{\prime \prime}$ & 968 & Silvânia & EPSPS \\
\hline POP6 & $\mathrm{S} 16^{\circ} 32^{\prime} 28^{\prime \prime} \mathrm{W} 48^{\circ} 38^{\prime} 16^{\prime \prime}$ & 941 & Gameleira de Goiás & EPSPS/ACCase \\
\hline POP7 & S $16^{\circ} 42^{\prime} 20^{\prime \prime} \mathrm{W} 48^{\circ} 34^{\prime} 37^{\prime \prime}$ & 925 & Silvânia & EPSPS/ACCase \\
\hline
\end{tabular}

${ }^{1 / H i s t o ́ r i c o ~ d o ~ c o n t r o l e ~ q u i ́ m i c o ~ n a s ~ u ́ l t i m a s ~ c i n c o ~ s a f r a s ~ a g r i ́ c o l a s . ~ A p l i c a c ̧ a ̃ o ~ d e ~ i n i b i d o r e s ~ d a ~ e n z i m a ~ 5-e n o l p i r u v i l s h i k i m a t e-3-f o s f a t o ~}$ sintase (EPSPs) e,ou, inibidores de acetil-CoA carboxilase (ACCase). 
um dendrograma de susceptibilidade ao herbicida glyphosate por meio da análise de agrupamento hierárquico de aglomeração da ligação média entre grupos ou UPGMA (Unweighted Pair Group Method with Arithmetic Mean) com base na distância euclidiana. O método de Mojena (1997) foi utilizado para determinação do número ótimo de grupos no dendrograma. As análises estatísticas foram efetuadas no software $\mathrm{R}^{\circledR}$ (R Development Core Team, 2017).

\section{RESULTADOS E DISCUSSÃO}

O teste "lack of fit" mostrou que não houve significância ( $p>0,05$; teste F) entre os resíduos do modelo de regressão não linear e os resíduos da ANOVA (fatorial) para controle e matéria seca (Tabela 2). Neste caso, os dados (do controle e da matéria seca) podem ser descritos adequadamente pela regressão não linear. Os parâmetros $a, b$ e $c$ das regressões log-logísticas para controle, aos 28 DAA, e para matéria seca, aos 56 DAA, podem ser observados na Tabela 3.
Com as curvas de dose-resposta traçadas para todas as populações, observou-se aumento de eficácia no controle do capim-amargoso com o incremento da dose de glyphosate (Figura 1 e 2). No entanto, as populações 3 e 5 foram menos afetadas pelo glyphosate do que as demais populações, visto que o incremento no controle ou na redução da matéria seca foi obtido apenas com maiores doses, a partir de $1,68 \mathrm{~kg} \mathrm{ha}^{-1}$.

A dose requerida de glyphosate $\left(\mathrm{kg} \mathrm{ha}^{-1}\right)$ para promover $50 \%$ de controle $\left(\mathrm{C}_{50}\right)$ de capim-amargoso, aos 28 DAA, foi de $0,170,0,154$ e 0,096, respectivamente, para as populações 2, 4 e 7, mais susceptíveis; 0,376 e 0,308 para populações 1 e 6, de susceptibilidade intermediária; 1,48 e 1,32 para as populações 3 e 5 , menos susceptíveis ao referido herbicida (Tabela 4). Com exceção das populações 3 e 5 , todas as populações revelaram menor $\mathrm{C}_{50}$ inferior a $0,4 \mathrm{~kg}$ $\mathrm{ha}^{-1} \mathrm{e}$, consequentemente, a dose de glyphosate que proporciona $80 \%$ de controle $\left(\mathrm{C}_{80}\right)$ foi menor que $1,68 \mathrm{~kg} \mathrm{ha}^{-1}$ (dose discriminatória). Diferentemente, nas populações 3 e 5 o $\mathrm{C}_{80}$ foi 3,36 e $3,73 \mathrm{~kg} \mathrm{ha}^{-1}$, respectivamente, valores

Tabela 2: Resultados do teste F para análise por falta de ajuste entre o modelo da ANOVA (fatorial) e a regressão não linear para controle, aos 28 DAA, e matéria seca, aos 56 DAA, em sete populações de capim-amargoso

\begin{tabular}{lccrrr}
\hline \multirow{2}{*}{ Resíduos combinados } & \multicolumn{2}{c}{ Controle } & & \multicolumn{2}{c}{ Matéria seca } \\
\cline { 2 - 3 } \cline { 5 - 6 } & gl & Soma dos quadrados & & gl & Soma dos quadrados \\
\hline Regressão não linear & 259 & 18635 & & 25926853 \\
ANOVA (fatorial) & 210 & 14058 & & 21024007 \\
Teste Lack-of-fit & 49 & $1,3955^{\mathrm{ns}}$ & & $490,5088^{\mathrm{ns}}$ \\
\hline
\end{tabular}

Tabela 3: Resumo dos parâmetros da curva dose-resposta para controle, aos 28 DAA, e matéria seca, aos 56 DAA, em sete populações de capim-amargoso

\begin{tabular}{|c|c|c|c|c|}
\hline \multirow{2}{*}{ População } & \multirow{2}{*}{ Variável } & \multicolumn{3}{|c|}{ Parâmetros da curva ${ }^{1 /}$} \\
\hline & & $a \pm$ e.p. & $b \pm$ e.p. & $c \pm$ e.p. \\
\hline \multirow{2}{*}{ POP 1} & Controle & $100,847 \pm 3,45$ & $0,376 \pm 0,04$ & $-1,043 \pm 0,14$ \\
\hline & Matéria seca & $100,720 \pm 5,01$ & $0,202 \pm 0,03$ & $0,973 \pm 0,13$ \\
\hline \multirow{2}{*}{ POP 2} & Controle & $100,491 \pm 1,73$ & $0,170 \pm 0,01$ & $-2,780 \pm 0,38$ \\
\hline & Matéria seca & $99,644 \pm 5,11$ & $0,139 \pm 0,01$ & $2,310 \pm 0,39$ \\
\hline \multirow{2}{*}{ POP 3} & Controle 28 & $100,379 \pm 3,57$ & $1,484 \pm 0,14$ & $-1,698 \pm 0,22$ \\
\hline & Matéria seca & $97,859 \pm 3,32$ & $1,038 \pm 0,08$ & $2,235 \pm 0,46$ \\
\hline \multirow{2}{*}{ POP 4} & Controle & $99,667 \pm 1,62$ & $0,154 \pm 0,01$ & $-4,928 \pm 0,69$ \\
\hline & Matéria seca & $100,030 \pm 5,09$ & $0,156 \pm 0,01$ & $6,255 \pm 1,30$ \\
\hline \multirow{2}{*}{ POP 5} & Controle & $105,276 \pm 3,61$ & $1,318 \pm 0,13$ & $-1,332 \pm 0,15$ \\
\hline & Matéria seca & $93,332 \pm 3,65$ & $1,035 \pm 0,12$ & $1,632 \pm 0,22$ \\
\hline \multirow{2}{*}{ POP 6} & Controle & $96,302 \pm 1,75$ & $0,308 \pm 0,02$ & $-5,703 \pm 0,92$ \\
\hline & Matéria seca & $92,789 \pm 4,14$ & $0,259 \pm 0,02$ & $3,942 \pm 0,86$ \\
\hline \multirow{2}{*}{ POP 7} & Controle & $99,868 \pm 1,65$ & $0,096 \pm 0,01$ & $-3,606 \pm 1,35$ \\
\hline & Matéria seca & $88,617 \pm 5,09$ & $0,102 \pm 0,01$ & $3,984 \pm 1,80$ \\
\hline
\end{tabular}

${ }^{1 /} \mathrm{Y}=\left\{\left[\mathrm{a} /\left[1+(\mathrm{x} / \mathrm{b})^{c}\right]\right\}\right.$, em que $\mathrm{Y}=$ variável resposta, $a=$ amplitude existente entre o ponto máximo e o mínimo da variável; $b=$ dose que promove uma resposta de $50 \%$ da variável; $c=$ inclinação da linha; e $x=$ dose do herbicida $\left(\mathrm{g} \mathrm{ha}^{-1}\right)$.

Dados são: média \pm erro padrão de quatro repetições. 
duas vezes maiores que a dose discriminatória, confirmando-as como as menos susceptíveis ao glyphosate.

Os resultados referentes à redução de 50 e $80 \%$ da matéria seca $\left(\mathrm{GR}_{50}\right.$ ou $\left.\mathrm{GR}_{80}\right)$ corroboraram os valores obtidos para $\mathrm{ED}_{50}$ e $\mathrm{ED}_{80}$, confirmando, nas populações 3 e 5 , a predominância de indivíduos menos sensíveis ao glyphosate. Por outro lado, nas demais populações foram identificadas doses de $\mathrm{GR}_{80}$ variando entre 0,14 e $0,84 \mathrm{~kg}$ ha $^{-1}$, determinando-as, portanto, como susceptíveis ao herbicida glyphosate.

As diferenças do nível de susceptibilidade, entre as populações de capim-amargoso, ao glyphosate, foi consequência do histórico do controle químico adotado nas diferentes áreas agrícolas (Tabela 1). Nesse sentido, as populações 3 e 5 (menos susceptíveis ao glyphosate) foram oriundas de áreas com manejo mais intensivo, em que esse herbicida foi a única estratégia adotada para controle do capim-amargoso. Ao contrário, as populações susceptíveis ao glyphosate (1, 2, 4, 6 e 7) foram provenientes de áreas onde se recomenda, há pelo menos cinco safras, a utilização de graminicidas (inibidores da acetil-CoA carboxilase), associados ao glyphosate como estratégia de controle.

Assim, os resultados desta pesquisa confirmam que a adoção de glyphosate como exclusiva estratégia de controle, aliada a aplicações sequenciais dessa mesma molécula herbicida tendem a selecionar populações de capimamargoso menos susceptíveis ao herbicida. Ressalva-se

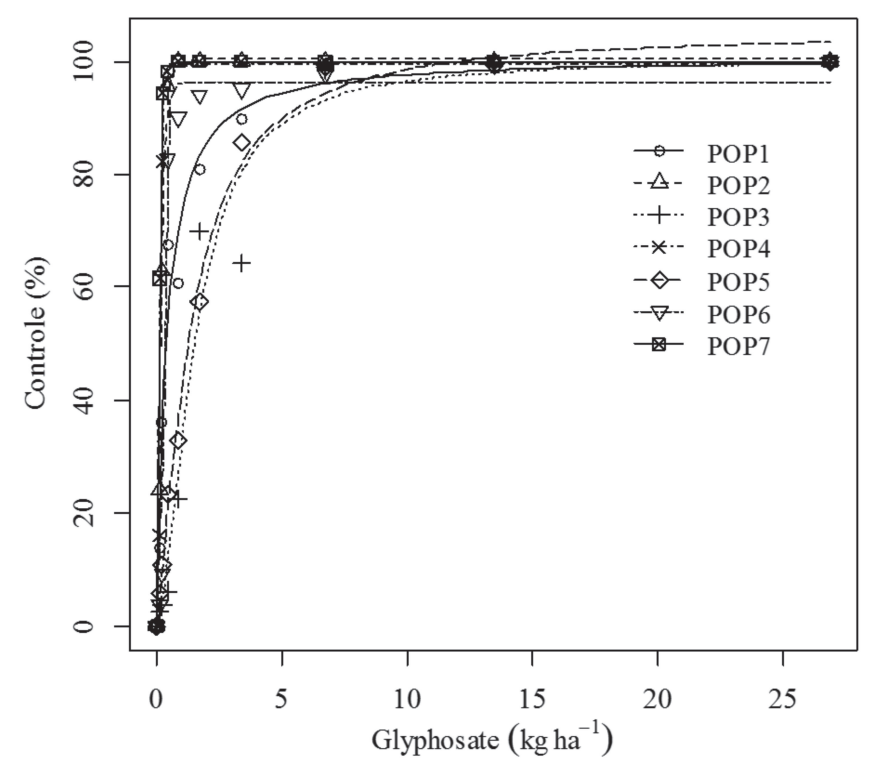

Figura 1: Curvas de resposta a doses de glyphosate para controle, aos 28 DAA, em sete populações de capim-amargoso.

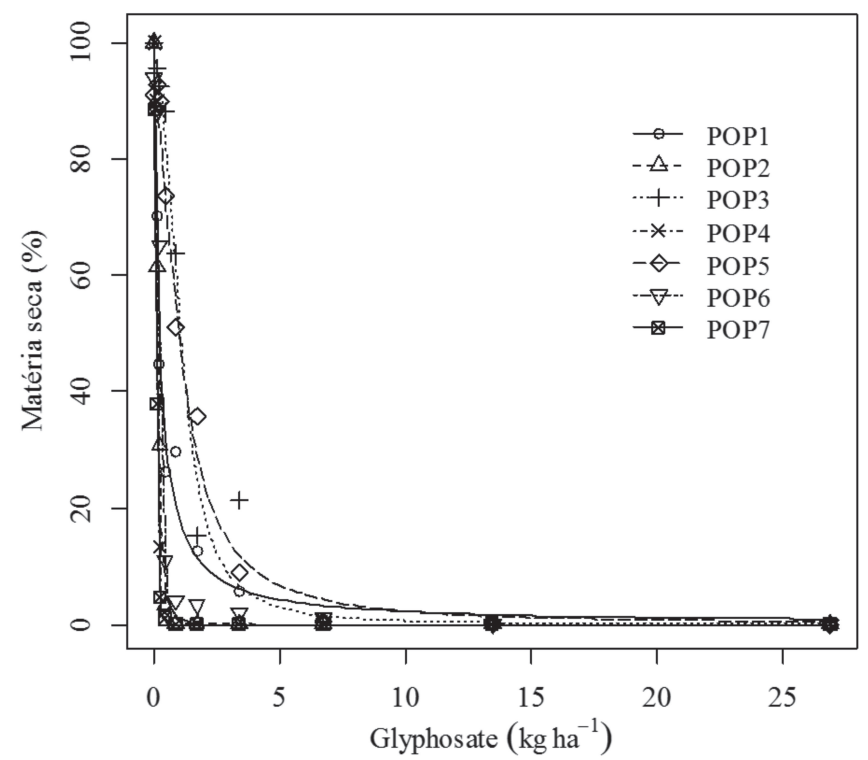

Figura 2: Curvas de resposta a doses de glyphosate para matéria seca da parte aérea aos 56 DAA, em sete populações de capimamargoso.

Rev. Ceres, Viçosa, v. 66, n.1, p. 018-025, jan/fev, 2019 
que em todas as áreas não havia histórico de cultivo de milho tolerante ao glyphosate. Logo, a alteração da susceptibilidade ao glyphosate, constatada nas populações 3 e 5, foi adquirida ao longo do tempo, em função do uso repetitivo do herbicida na cultura da soja, em decorrência do emprego de doses elevadas e do controle ineficaz nos estádios avançados de desenvolvimento das plantas de D. insularis.

Outros estudos também constataram a variabilidade de resposta das populações de capim-amargoso ao glyphosate, o que reforça a seleção de populações, prevalecendo no campo as menos afetadas pelo herbicida (Correia et al., 2010, Correia et al., 2015, Gomes et al., 2017).

Uma das possíveis causas da susceptibilidade diferencial ao herbicida glyphosate entre as populações de capim-amargoso está relacionada com as alterações nos processos de absorção, translocação, metabolismo e mutação genética da enzima EPSPS (Carvalho et al., 2012). A alteração no local de ação é apontada como mecanismo de resistência de D. insularis ao glyphosate (Galeano et al., 2016). Também foi relatado, por Barroso et al. (2015), que mudanças na morfologia e anatomia das folhas podem interferir na absorção do glyphosate e, portanto, na eficácia de controle do capim-amargoso.

Além disso, o controle eficiente do capim-amargoso em estádio avançado de desenvolvimento torna-se mais difícil, principalmente pela formação dos rizomas, o qual permite a rebrota das plantas (Machado et al., 2006). Por isso, avaliou-se a rebrota aos 56 dias após o corte (DAC) da parte aérea das sete populações, em função das doses de glyphosate. O teste "lack of fit" não foi significativo ( $>00,05$; teste qui-quadrado) para os dados de rebrota, logo podem ser descritos pela análise de regressão não linear. Os parâmetros $b$ e $c$ da equação log-logística encontram-se na Tabela 5.

As populações mais susceptíveis (2, 4 e 7$)$ tiveram $50 \%$ da rebrota inibida com doses de 0,15 a $0,24 \mathrm{~kg} \mathrm{ha}^{-1}$ de glyphosate, enquanto as populações de susceptibilidade intermediária (1 e 6) com doses de 0,81 a $0,87 \mathrm{~kg} \mathrm{ha}^{-1}$, respectivamente. Por outro lado, para a supressão de $50 \%$ da rebrota das populações menos susceptíveis (3 e 5), foram necessárias doses superiores a 2,0 $\mathrm{kg} \mathrm{ha}^{-1}$ de glyphosate (Tabela 4). Essas diferenças na capacidade de rebrota das populações de $D$. insularis também foram atribuídas às diferenças na susceptibilidade ao glyphosate.

A rebrota das plantas foi inibida totalmente conforme o aumento das doses de glyphosate, independentemente da susceptibilidade das populações (Figura 3). Todavia, até a dose de 1,68 $\mathrm{kg} \mathrm{ha}^{-1}$ de glyphosate (dose discriminatória), observou-se a rebrota das populações 1 e 6 , de susceptibilidade intermediária e, sobretudo, das populações 3 e 5, as menos susceptíveis ao glyphosate. Os resultados indicam que a dose de $1,68 \mathrm{~kg} \mathrm{ha}^{-1}$ de glyphosate não impediu a formação de rizomas, que após o corte da parte aérea permitiu a rebrota das plantas nessas quatro populações.

Timossi et al. (2006) constataram rápida rebrota em plantas perenizadas de capim-amargoso, mesmo quando tratadas com 1,44 $\mathrm{kg} \mathrm{ha}^{-1}$ de glyphosate. Este fato está

Tabela 5: Resumo dos parâmetros da curva dose-resposta para rebrota, aos 56 DAC, em sete populações de capim-amargoso

\begin{tabular}{lcc}
\hline \multirow{2}{*}{ População } & \multicolumn{2}{c}{ Parâmetros da curva $^{\mathbf{1 / N s}}$} \\
\cline { 2 - 3 } & $\boldsymbol{b} \pm$ e.p. & $\boldsymbol{c} \pm$ e.p \\
\hline POP 1 & $1,216 \pm 0,38$ & $0,814 \pm 0,32$ \\
POP 2 & $5,350 \pm 2,99$ & $0,237 \pm 0,04$ \\
POP 3 & $1,326 \pm 0,41$ & $2,198 \pm 0,81$ \\
POP 4 & $2,650 \pm 1,34$ & $0,158 \pm 0,04$ \\
POP 5 & $0,929 \pm 0,30$ & $2,479 \pm 1,14$ \\
POP 6 & $1,031 \pm 0,33$ & $0,871 \pm 0,38$ \\
POP 7 & $2,188 \pm 1,09$ & $0,152 \pm 0,05$ \\
\hline
\end{tabular}

${ }^{1 /} \mathrm{Y}=\{[1 /[1+(\mathrm{x} / \mathrm{b})]\}$, em que $\mathrm{Y}=$ variável resposta; $\mathrm{b}=$ dose que promove uma resposta de $50 \%$ da variável; e $\mathrm{x}=$ dose do herbicida $\left(\mathrm{kg} \mathrm{ha}^{-1}\right)$.

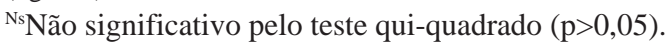

Dados são média \pm erro padrão de quatro repetições.

Tabela 4: Doses estimadas de glyphosate para controle $\left(\mathrm{C}_{50}\right)$ e redução de matéria seca da parte aérea $\left(\mathrm{GR}_{80}\right)$, em sete populações de capim-amargoso

\begin{tabular}{|c|c|c|c|c|}
\hline \multirow{2}{*}{ População } & $C_{50} \pm$ e.p. & $\mathrm{C}_{80} \pm$ e.p. & $\mathbf{G R}_{50} \pm$ e.p. & $\mathrm{GR}_{80} \pm$ e.p. \\
\hline & \multicolumn{4}{|c|}{ kg i.a. ha $^{-1}$} \\
\hline POP 1 & $0,376 \pm 0,05$ & $1,421 \pm 0,37$ & $0,203 \pm 0,03$ & $0,842 \pm 0,16$ \\
\hline POP 2 & $0,170 \pm 0,01$ & $0,279 \pm 0,02$ & $0,139 \pm 0,01$ & $0,253 \pm 0,03$ \\
\hline POP 3 & $1,484 \pm 0,14$ & $3,358 \pm 0,60$ & $1,038 \pm 0,08$ & $1,930 \pm 0,27$ \\
\hline POP 4 & $0,154 \pm 0,01$ & $0,204 \pm 0,01$ & $0,156 \pm 0,01$ & $0,194 \pm 0,01$ \\
\hline POP 5 & $1,318 \pm 0,13$ & $3,732 \pm 0,67$ & $1,035 \pm 0,12$ & $2,419 \pm 0,32$ \\
\hline POP 6 & $0,308 \pm 0,02$ & $0,393 \pm 0,02$ & $0,259 \pm 0,02$ & $0,368 \pm 0,04$ \\
\hline POP 7 & $0,096 \pm 0,01$ & $0,142 \pm 0,01$ & $0,102 \pm 0,01$ & $0,145 \pm 0,02$ \\
\hline
\end{tabular}

$\mathrm{C}_{50}$ ou $\mathrm{C}_{80}$ são doses necessárias para 50 ou $80 \%$ de controle e $\mathrm{GR}_{50}$ ou $\mathrm{GR}_{80}$ são doses necessárias para 50 e $80 \%$ de redução da matéria seca da parte aérea.

Dados são média \pm erro padrão de quatro repetições. 
relacionado com a grande quantidade de amido dos rizomas, que dificulta a translocação do glyphosate e permite a intensa rebrota da parte aérea, mesmo após tratada com o herbicida (Machado et al., 2008).

A susceptibilidade diferencial ao herbicida glyphosate também foi estudada por meio da análise de agrupamento hierárquico, obtendo-se um dendrograma pelo método UPGMA, a partir das medidas de dissimilaridade entre as populações de $D$. insularis, com base na distância euclidiana (Figura 4). A aplicação do método de Mojena sugeriu um corte na altura de $\theta=2,86$, discriminando dois grupos quanto à susceptibilidade ao glyphosate. O primeiro grupo foi composto pelas populações 3 e 5, enquanto o segundo grupo foi formado pelas demais populações (1, 2, 4, 6 e 7). Desse modo, as populações 3 e 5, consideradas as menos susceptíveis ao glyphosate, foram seme- lhantes entre si e, por isso integraram o mesmo grupo no dendrograma, ao passo que foram distintas das demais populações de capim-amargoso, as de susceptibilidade intermediária e as mais afetadas pelo glyphosate. Portanto, a análise de agrupamento mostrou-se eficaz na discriminação da sensibilidade das populações ao glyphosate. Logo, sugere-se a utilização da técnica para estudos, envolvendo a resistência de plantas daninhas a herbicidas.

Em síntese, foi confirmada a hipótese da susceptibilidade diferencial ao herbicida glyphosate, como consequência do histórico do controle químico, acarretando também diferenças na capacidade de rebrota das plantas. A associação, em tanque, de dois herbicidas com diferenças nos mecanismos de ação pode contribuir para redução da pressão de seleção imposta pelo glyphosate. Entretanto, a aplicação contínua pode não ser efetiva em todas as situações.

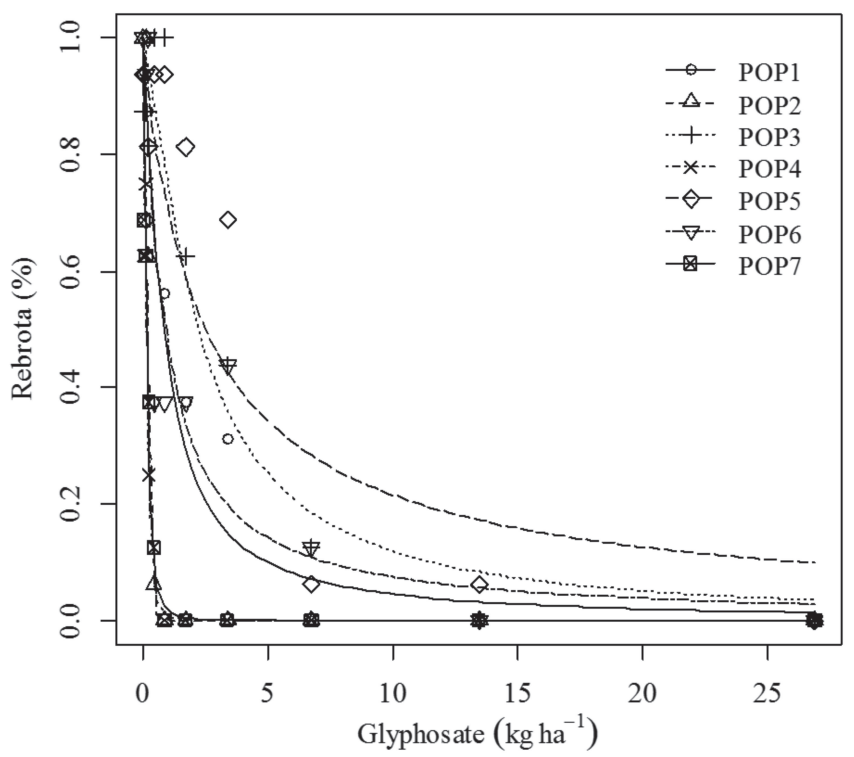

Figura 3: Curvas de resposta a doses de glyphosate para rebrota das plantas, aos 56 DAC, em sete populações de capim-amargoso.

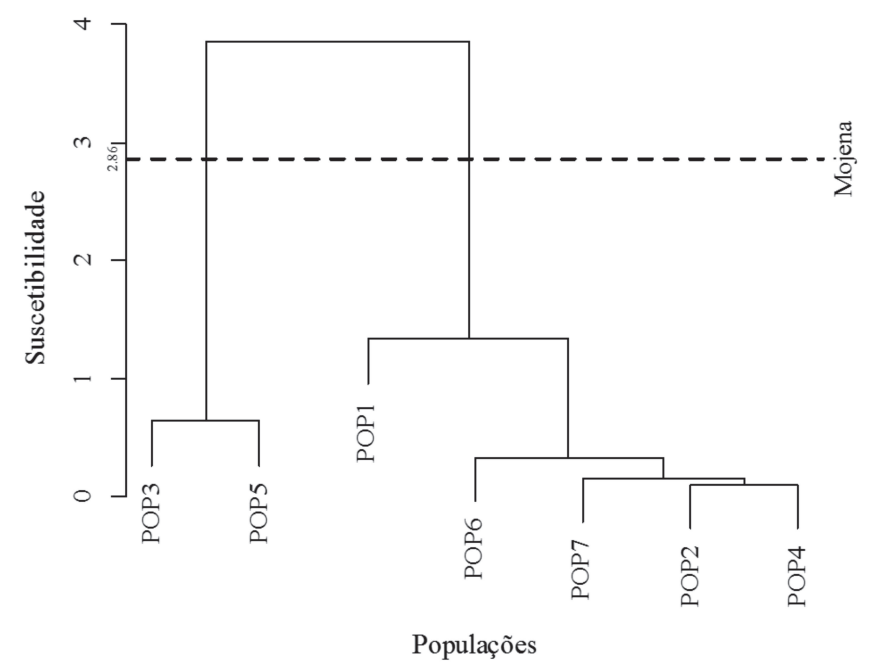

Figura 4: Dendrograma obtido pelo método UPGMA, a partir das medidas de dissimilaridade entre sete populações de capimamargoso ao herbicida glyphosate, com base na distância de euclidiana. 


\section{CONCLUSÕES}

As populações de capim-amargoso, manejadas há cinco anos com glyphosate associado aos herbicidas inibidores da ACCase, são susceptíveis ao glyphosate.

As populações de capim-amargoso com manejo mais intensivo unicamente com glyphosate são menos sensíveis a esse herbicida.

As populações de capim-amargoso menos susceptíveis ao glyphosate apresentam maior capacidade de rebrota da parte aérea, após tratadas com doses elevadas do herbicida.

\section{REFERÊNCIAS}

Barroso AAM, Galeano E, Albrecht AJP, Reis FC \& Filho RV (2015) Does Sourgrass leaf anatomy influence glyphosate resistance?. Comunicata Scientiae, 06:445-453.

Carvalho SJP, Lombardi BP, Nicolai M, López-Ovejero RF, Christoffoleti PJ \& Medeiros D (2005) Curvas de dose-resposta para avaliação do controle de fluxos de emergência de plantas daninhas pelo herbicida imazapic. Planta Daninha, 23:535-542.

Carvalho LB, Alves PLCA, González-Torralva F, Cruz-Hipolito HE, Rojano-Delgado AM, De Prado R, Gil-Humanes J, Barro F \& De Castro MD (2012) Pool of resistance mechanisms to glyphosate in Digitaria insularis. Journal of Agricultural and Food Chemistry, 60:615-622.

Céleres (2018) Projeção de safra 2017/2018: soja, março de 2018. Disponível em: http://www.celeres.com.br/ic18-01-projecao-desafra-soja-janeiro-2018/. Acessado em: 22 janeiro de 2018.

Conab - Companhia Nacional de Abastecimento (2018) Levantamento de safras. Disponível em: http://www.conab.gov. br/ conteudos.php?a=1252\&t=. Acessado em: 22 janeiro de 2018 .

Correia NM, Acra LT \& Balieiro G (2015) Chemical control of different Digitaria insularis populations and management of a glyphosate-resistant population. Planta Daninha, 33:93-101.

Correia NM, Leite GJ \& Garcia LD (2010) Resposta de diferentes populações de Digitaria insularis ao herbicida glyphosate. Planta Daninha, 28:769-776.

Embrapa - Empresa Brasileira de Pesquisa Agropecuária (2013) Sistema brasileiro de classificação de solos. Brasília, Embrapa Solos. 353p.

Galeano E, Barroso AAM, Vasconcelos TS, López-Rubio A, Albrecht AJP, Filho RV \& Carrer H (2016) EPSPS variability, gene expression, and enzymatic activity in glyphosate-resistant biotypes of Digitaria insularis. Genetics and Molecular Research, 15:01-15.

Gazziero DLP, Voll E, Fornarolli D \& Adegas LFS (2012) Efeitos da convivência do capim-amargoso na produtividade da soja. In: XXVIII Congresso Brasileiro da Ciência das Plantas Daninhas, Campo Grande. Anais, SBCPD. p.346-350.

Gemelli A, Oliveira Jr RS, Constantin J, Braz GBP, Jumes TMC, Gheno EA, Rios FA \& Franchini LHM (2013) Estratégias para o controle de capim-amargoso (Digitaria insularis) resistente ao glyphosate na cultura milho safrinha. Revista Brasileira de Herbicidas, 12:162-170.

Gomes LJP, Santos JI, Gasparino EC \& Correia NM (2017) Chemical control and morphoanatomical analysis of leaves of different populations of sourgrass. Planta Daninha, 35:01-11.
Green JM \& Owen MDK (2011) Herbicide-resistant crops: utilities and limitations for herbicide-resistant weed management. Journal Agricultural and Food Chemistry, 59:5819-5829.

Heap I (2018) The international survey of herbicide resistance. Disponível em: www.weedscience.com/summary/home.aspx. Acessado em: 15 janeiro de 2018.

Lopez Ovejero RF, Takano HK, Nicolai M, Ferreira A, Melo MSC, Cavenaghi AL, Christoffoleti PJ \& Oliveira Jr RS (2017) Frequency and dispersal of glyphosate-resistant sourgrass (Digitaria insularis) populations across brazilian agricultural production areas. Weed Science, 65:285-294.

Lorenzi H (2014) Manual de identificação e controle de plantas daninhas: plantio direto e convencional. $7^{\mathrm{a}}$ ed. Nova Odessa, Instituto Plantarum. 383p.

Machado AFL, Ferreira LR, Ferreira FA, Fialho CMT, Tuffi Santos LD \& Machado MS (2006) Análise de crescimento de Digitaria insularis. Planta Daninha, 24:641-647.

Machado AFL, Meira RMS, Ferreira LR, Ferreira FA, Tuffi Santos LD, Fialho CMT \& Machado MS (2008) Caracterização anatômica de folha, colmo e rizoma de Digitaria insularis. Planta Daninha, 26:01-08.

Mojena R (1977) Hierarchical grouping methods and stopping rules: an evaluation. The Computer Journal, 20:359-363.

$\mathrm{R}$ development core team (2017) R: A Language and environment for statistical computing. Disponível em: http://www.Rproject.org. Acessado em: 21 de abril de 2017.

Ritz C, Kniss AR \& Streibig JC (2015) Research methods in weed science: statistics. Weed Science, 63:166-187.

Shaner DL (2000) The impact of glyphosate-tolerant crops on the use of other herbicides and on resistance management. Pest Management Science, 56:320-326.

SBCPD - Sociedade Brasileira da Ciência das Plantas Daninhas (1995) Procedimentos para instalação, avaliação e análise de experimentos com herbicidas. Londrina, SBCPD. 42p.

Timossi PC, Durigan JC \& Leite GJ (2006) Eficácia de glyphosate em plantas de cobertura. Planta Daninha, 24:475-480. 